\title{
Effects of Relationship with Adult Children on Mental Health of the Elderly in China
}

\author{
Junran Jia ${ }^{1, \mathrm{a}}$ \\ ${ }^{1}$ Raffles Institution, ${ }^{1}$ Raffles Institution Ln, Singapore 575954 \\ ajasmineea0088@gmail.com \\ *Junran Jia
}

\begin{abstract}
.
To study how a relationship with the adult and children relate to the mental health of the elderly in China, we draw unique panel data from the China Health and Retirement Longitudinal Study (CHARLS). By using the 2015 wave which consists of 21095 Chinese residents ages 45 and older and 12235 households, we construct a regression model using the number of times of visit in a year and total support from children as independent variables and depression rate as the dependent variable. The regression analysis reveals that greater times of visit by children is associated with lower depression rate. Monetary support and in-kind support, however, do not necessarily result in better mental health condition. These findings provide some evidence on the importance of children accompany on the mental health of the elderly, which is supported by the results of other related research. Discussions on further implication of the results from both the individual and society's perspective are also included as a suggestion for future improvement.
\end{abstract}

Keywords: mental health, elderly, visit, regression model

\section{Introduction}

The world's population is ageing rapidly. Between 2015 and 2050 , the proportion of the world's older adults is estimated to almost double from about $12 \%$ to $22 \%$. In absolute terms, this is an expected increase from 900 million to 2 billion people over the age of 60 . Specifically, China is ageing more rapidly than almost any country in recent history: In 2015, $9.5 \%$ of the population of China was aged 65 or older and this percentage is projected to increase to $27.5 \%$ by 2050 . (Rapoza K, 2017)

Older people face special physical and mental health challenges which need to be recognized. Over $20 \%$ of adults aged 60 and over suffer from a mental or neurological disorder (excluding headache disorders) and $6.6 \%$ of all disability (disability adjusted life years-DALYs) among people over 60 years is attributed to mental and neurological disorders. These disorders in older people account for $17.4 \%$ of Years Lived with Disability (YLDs). The most common mental and neurological disorders in this age group are dementia and depression, which affect approximately 5\% and $7 \%$ of the world's older population, respectively. Anxiety disorders affect $3.8 \%$ of the older population, substance use problems affect almost $1 \%$ and around a quarter of deaths from self-harm are among people aged 60 or above. Anxiety disorders are highly comorbid with depression in older adults and are highly comorbid with a number of medical illnesses. Associations between cognitive decline and anxiety have also been observed. (Kate B. et al,2010)
Mental health problems are under-identified by health-care professionals and older people themselves, and the stigma surrounding these conditions makes people reluctant to seek help. According to a previous research (Xu et al, 2017), the weighed 1-month prevalence of mental disorders for elderlies was $14.27 \%$, whereas the lifetime prevalence of mental disorders was $24.20 \%$, which is one in every five elderlies. (i.e. Period prevalence is defined as the proportion of a population that has the condition during a given period of one month or one year in this case). Unfortunately, with globalization and rapid economic growth in China in recent years, working age population is living an increasingly busy and fast-paced life. 996 working hour system is a de facto work schedule practiced in the People's Republic of China. It derives its name from its requirement that employees work from 9:00 am to 9:00 pm, 6 days per week. A number of Chinese Internet companies have adopted this system as their official work schedule. To seek a better job opportunity, a large proportion of young adults live in different cities from their elderly parents, but this would mean that they have much lesser time to spend with the elderly. In fact, the percentage of elderly living alone has increased from $42 \%$ to $54 \%$ from 2000 to 2010 , while the increase in rural areas was from $37.9 \%$ to $45.6 \%$. According to Chinese state-run news agency Xinhua(http://www.xinhuanet.com/), China has 28000 registered nursing institutes for the elderly and more than 7 million elderly home beds, which has more than tripled itself in the past five years. This shows from a demand-side point of view that more and more elder people choose to live in nursing homes and to be taken care of by nursing workers instead of by their children. 
Being one of the most debated issues in China currently, the increasing distance between elderlies and their children would give rise to many questions. Will the lack of company of their children lead to an undesirable period of time for the elderly? How does the relationship between the elderly and their adult children affect the emotion of the elderly? What kind of negative emotion will this bring about? How far does it cause depression to the elderly? Many researches are done to find out how social interactions can affect the elderly's mental health, but this research would focus specifically on how interaction with their children affects the emotion of the elderly. The trend is that the issue of mental illnesses will only increase at an ever-increasing rate if no measure is implemented. Being a major issue in China's current society, the problem of lack of support and company from the children definitely requires deeper attention. The effect of elderly-children relationship on the elderly's mental well-being is discussed and analyzed in this paper.

We start this paper with introduction and summary of statistics of the data used in this study. It is followed by the regression model used to uncover the relationship between elderly-children relationship and the mental health of the elderly. This is then followed by detailed description of the key variables we use in the study and their distribution is shown using figures. After that, various graphs showing relationship between the key X-variable and Y-variable are presented, followed by the regression results presented in a table. We further discuss the implication of these results and conclude this paper with a summary of all data and results in the study.

\section{DATA}

This study uses data from China Health and Retirement Longitudinal Study (CHARLS), which is a nationwide survey conducted by Peking University. It aims to collect a high quality nationally representative sample of Chinese residents ages 45 and older to serve the needs of scientific research on the elderly. The baseline national wave of CHARLS is being fielded in 2011 and includes about 10000 households and 17500 individuals in 150 counties/districts and 450 villages/resident committees in 28 provincial administrative unit in mainland China. The individuals are followed up every two years using face-to-face, computer-aided personal interviews (CAPI).

CHARLS is based on the Health and Retirement Study (HRS) and related aging surveys such as the English Longitudinal Study of Aging (ELSA) and the Survey of Health, Aging and Retirement in Europe (SHARE). CHARLS adopts multi-stage stratified PPS (Probability Proportional to Size) sampling. Within each province, CHARLS randomly selected 13 county level units (rural counties or urban districts) by PPS (Probability Proportional to Size), stratified by regions and urban/rural. The goal was to achieve a sample size of 16 households in each village level unit using a complete list of dwelling units generated from a map for each province. CHARLS did not allow for sample replacement for fear of causing interviewers to non-randomly select households to interview. Instead, a pre-determined number of households were interviewed in every village. The total sample size of 2015 wave is 21095 individuals in 12235 households.

The CHARLS questionnaire includes the following modules: demographics, family structure/transfer, health status and functioning, biomarkers, health care and insurance, work, retirement and pension, income and consumption, assets (individual and household). In addition to the typical HRS survey content, a detailed community-level questionnaire was formulated. This community questionnaire focuses on important infrastructure available in the community, plus on the availability of health facilities used by the elderly and on prices of goods and services often used by the elderly.

The sampling protocol is that if one member of the household of age 45 and over is sampled and their spouse (no matter what the spouse's age is) will also be sampled. The analysis in this paper uses the 2015 wave of the CHARLS national survey, which is the most updated data, thus, could reflect the latest understanding of the relationship between elderlies-children relationship and elderly's mental well-being. Since the scope of this study is mental health of the elderly in China, so we only include those that are at least 45 years old in the analysis and there are 20078 of them.

The detailed construction of the sample used in the analysis is shown in the table below.

Table 1. Database Procedure Table

\begin{tabular}{llc}
\hline Drop & Description & Remain \\
\hline & Merge & 23353 \\
3998 & Drop obs with missing key variable depression & 19355 \\
59 & Drop obs with missing age & 19296 \\
914 & Keep obs with age $>45$ & 18382 \\
1 & Drop obs with missing gender & 18381 \\
13 & Drop obs with misssing marriage info & 18368 \\
1576 & Drop obs with missing education years & 16792 \\
8300 & Drop obs with missing expenditure info & 8492 \\
320 & Drop obs with missing pension info & 8172 \\
944 & Drop obs with missing health insurance info & 7228 \\
10 & Drop obs with missing smoking or drinking info & 7218 \\
1056 & Drop obs with missing height or weight info & 6162 \\
116 & obs with missing key variable total_support & 6046 \\
490 & obs with missing key variable visit & 5556 \\
\hline
\end{tabular}




\section{METHODS}

We utilize the following two regression models to examine the relationship between elderly-children relationship and the mental health of the elderly.

Mental Health $_{i}=\beta_{0}+\beta_{\text {Isupport }_{i}}+\beta_{2 \text { male }_{i}}+\beta_{3}$ age $_{i}+$ $\beta_{4 \text { schooling }_{i}}+\beta_{\text {surban }_{i}}+\beta_{\text {smarried }_{i}}+\beta_{\text {7pension }_{i}}+$ $\beta_{\text {sexpenditure }_{i}}+\beta$ ghealth_insurance $_{i}+\beta_{10}$ smoking $_{i}+$ $\beta_{11}$ drinking $_{i}+\beta_{12} B M I_{i}+u_{i}(1)$

Mental Health $_{i}=\beta_{0}+\beta_{\text {Ivist }_{i}}+\beta_{\text {2male }_{i}}+\beta_{3}$ age $_{i}+$ $\beta_{4 \text { Schooling }_{i}}+\beta_{\text {surban }_{i}}+\beta_{6}$ married $_{i}+\beta_{7 \text { pension }_{i}}+$ $\beta_{\text {sexpenditure }_{i}}+\beta_{\text {g health_insurance }}+\beta_{10}$ smoking $_{i}+$ $\beta_{11}$ drinking $_{i}+\beta_{12} B M I_{i}+u_{i}(2)$

Scores of two items are calculated as the key independent variables of the study. The first independent variable is frequency of visit by child, defined by question CD003
"How often do you see (child's name)?)". This item is assessed by a four-point-scale, scored as follows: $1=$ more than once a week, $2=$ more than once every 3 months, $3=$ more than once a year, $4=$ almost never. We recode the variable to assess it in terms of number of times that the respondents see their children by summing up the total number of days the respondent see each of the children in a year Another independent variable is total support from children, which comprises of money support and in-kind support and is defined by question CE009 "In the past one year, how much economic support did you and your spouse receive from your children?" This variable is measured in terms of monetary value as a sum of both money and in-kind support.

The two key $\mathrm{x}$ variables constructed are shown in the figures below.

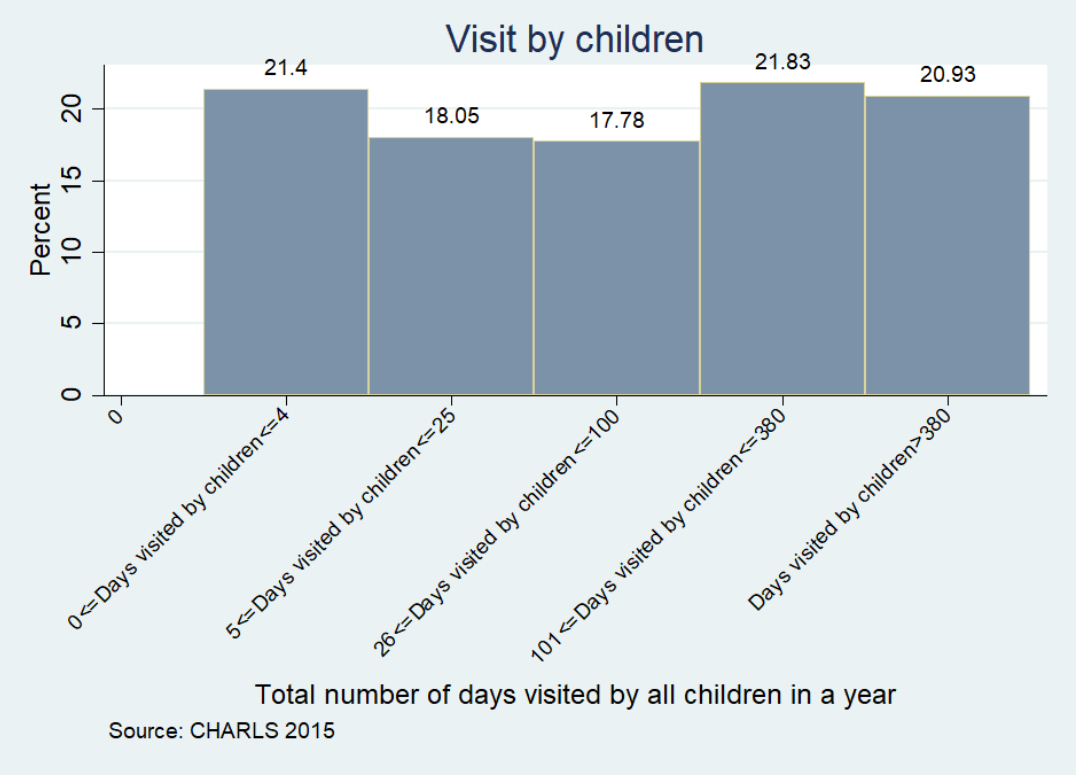

Fig. 1. Total number of days visited by all children in a year. In this histogram, $x$-axis represents the dependent variable, Total number of days visited by all children in a year, and y-axis represents the percentage of respondents. Frequency of visit by child, defined by question CD003 "How often do you see (child's name)?)" is assessed by a four-point-scale, scored as follows: 1=more than once a week, $2=$ more than once every 3 months, $3=$ more than once a year, $4=$ almost never. In our study, we recode it into actual number of days that respondents see their children and group them to construct this graph. There are 5556 observations for this variable.

Figure 1 reflects the total number of days the respondents see their children in a year. Maximum number $(21.83 \%)$ of respondents are visited by their children between 101 and 380 days in the past one year. Besides, $21.4 \%, 18.05 \%$,
$17.38 \%$ and $20.93 \%$ of respondents are visited by their children for a total number of less than four times, between 5 and 25 times, between 26 and 100 times and more than 380 times in the past one year respectively. 


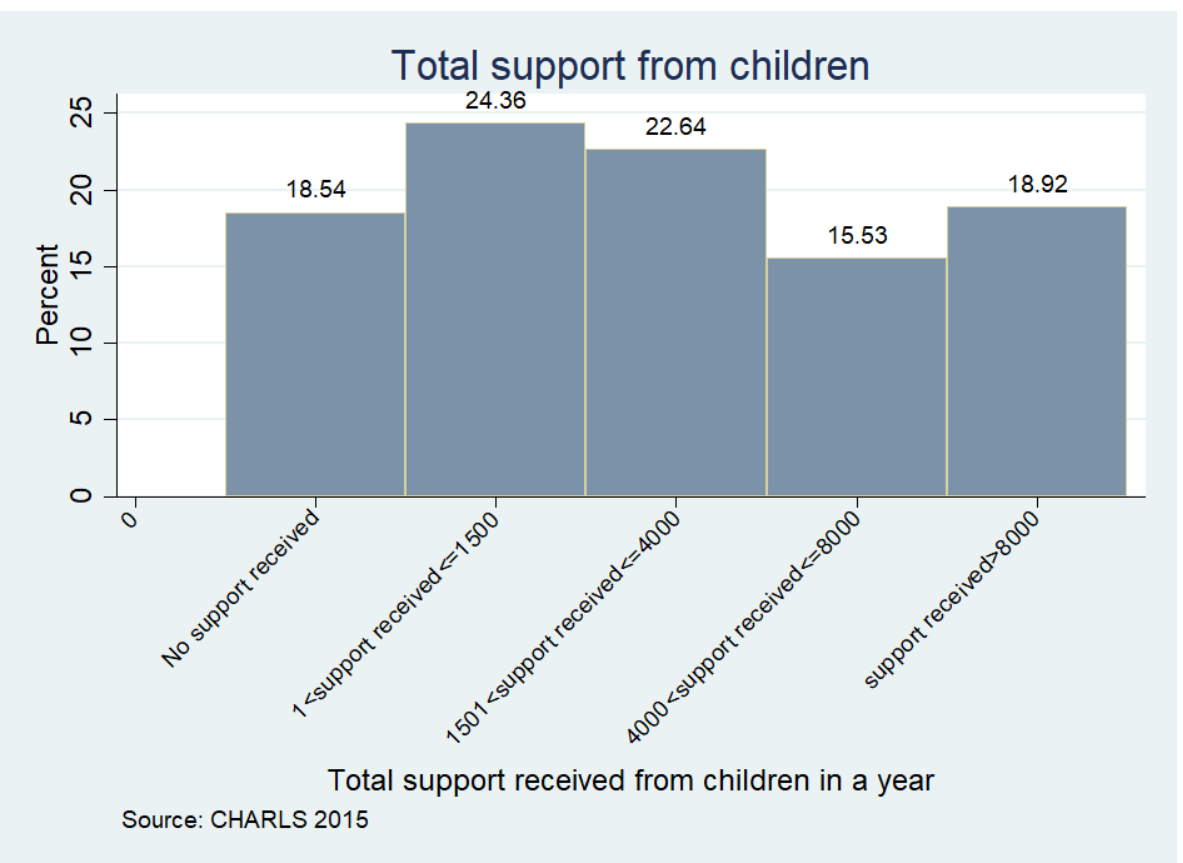

Fig. 2. Self-reported depression of the Respondents. In this histogram, $\mathrm{x}$-axis represents the dependent variable, total support received from children in a year, and y-axis represents the percent of respondents. The dependent variable, total support from children, comprises of money support and in-kind support and is defined by question CE009 "In the past one year, how much economic support did you and your spouse receive from your children?" It is measured in terms of monetary value but in this case, we group them up to plot this graph. There are 6046 observations for this variable.

Figure 2 reflects total support respondents receive from their children in the past one year. About one quarter of respondents $(24.36 \%)$ received amount between 1 and 1500 yuan and $22.64 \%$ of the respondents received between 1501 and 4000 yuan. Higher value of 4000 to 8000 and 8000 to maximum are received by $15.53 \%$ and $18.92 \%$ of respondents respectively. $18.54 \%$ reported zero support received.
The key dependent variable is defined as degree of depression. It is defined based on question DC011 "The frequency that I felt depressed". It is assessed by a four-point scale, scored as follows: $1=$ Rarely or none of the time ( $<1$ day), $2=$ Some or a little of the time (1-2 days), $3=$ Occasionally or a moderate amount of the time (3-4 days), and $4=$ Most or all of the time (5-7 days) out of a week. 


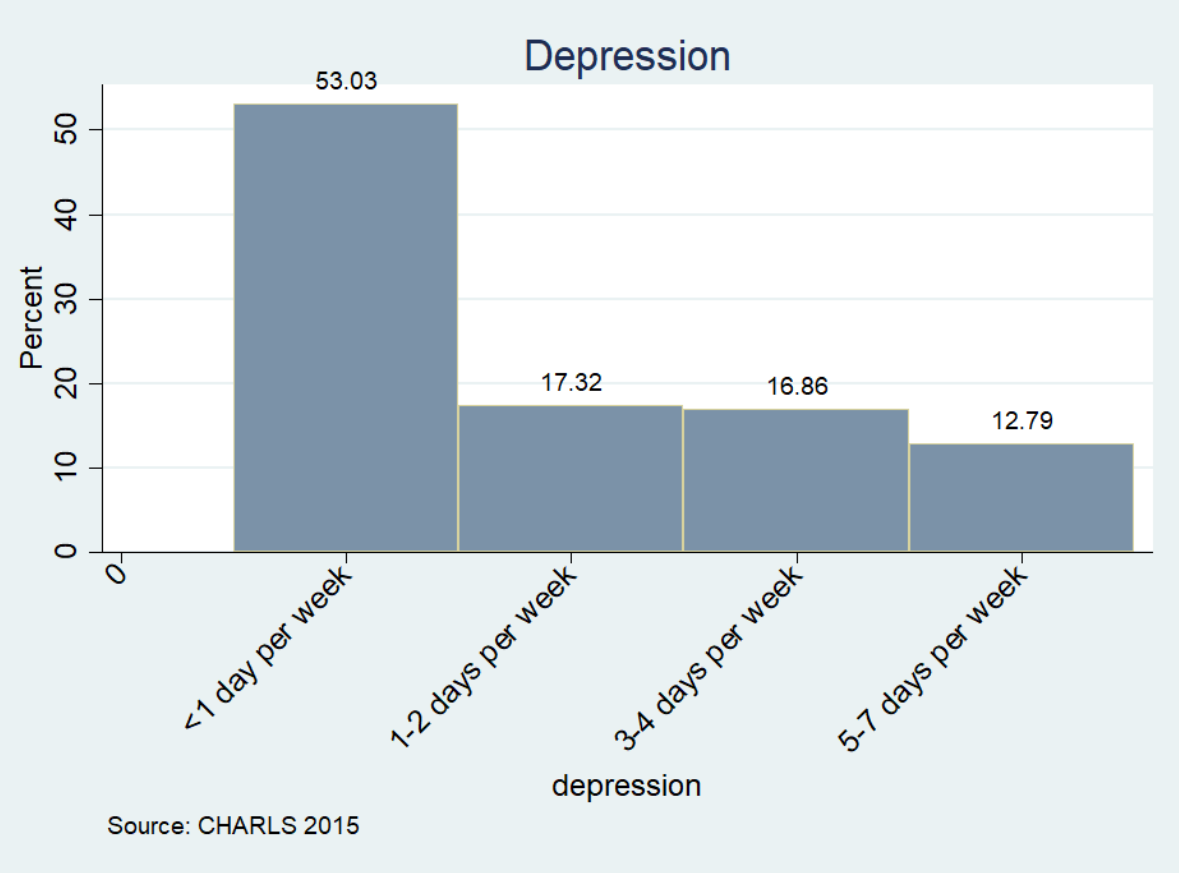

Fig. 3. Self-reported depression of the Respondents. In this histogram, $x$-axis represents the dependent variable, level of depression, and y-axis represents the percent of respondents. Depression is defined based on question DC011 "I felt depressed". It is assessed by a four-point scale, scored as follows: $1=$ Rarely or none of the time ( $<1$ day), $2=$ Some or a little of the time $(1-2$ days), 3 = Occasionally or a moderate amount of the time (3-4 days), and $4=$ Most or all of the time (5-7 days) out of a week. There are 5556 observations for this variable.

Figure 3 reflects the level of depression of CHARLS respondents. Slightly more than half of the respondents $(53.03 \%)$ reported feeling depressed for less than a day per week, and $17.32 \%, 16.86 \%$ and $12.79 \%$ of the respondents reported depression for 1-2 days, 3-4 days and 5-7 days per week respectively. This indicates that half of the respondents experience good emotion while others experience varying levels of depression.

We include demographical variables including male, age, urban and educational level, which are shown in the demographic information table. The variable "male" measures gender as 1 represents male and 0 represents female. Ages are calculated based on their self-reported date of birth as well as information on ID based on time of survey which is June 2015. Address is measured by variable "urban" as 1 represents urban address and 0 represents rural address. Education level are based on question bd001 w2 4 "What is your highest education level attained?" and measured according to years of schooling. Demographic variables are listed in Table 2.

Table 2. Demographic Table

\begin{tabular}{llllllllll}
\hline \multirow{2}{*}{$\begin{array}{l}\text { Age } \\
\text { group }\end{array}$} & Gender & \multicolumn{3}{c}{ Education } & \multicolumn{3}{c}{ Address Type } \\
\cline { 2 - 10 } & Male/\% & Female/\% & Illiterate/\% & $\begin{array}{c}\text { <elementary } \\
\text { school/\% }\end{array}$ & $\begin{array}{c}\text { elementary } \\
\text { school/\% }\end{array}$ & $\begin{array}{c}\text { middle } \\
\text { school/\% }\end{array}$ & $\begin{array}{c}\text { >High } \\
\text { school/\% }\end{array}$ & Rural/\% & Urban/\% \\
\hline $45-49$ & 5.114 & 11.351 & 4.185 & 8.347 & 10.998 & 9.506 & 6.218 & 8.333 & 7.563 \\
$50-54$ & 15.712 & 22.462 & 8.072 & 12.602 & 17.379 & 32.424 & 26.813 & 18.06 & 20.495 \\
$55-59$ & 15.434 & 16.221 & 14.798 & 12.52 & 10.523 & 18.276 & 28.497 & 16.119 & 15.219 \\
$60-64$ & 20.579 & 17.593 & 22.197 & 23.404 & 17.447 & 16.433 & 15.285 & 19.303 & 18.908 \\
$65-69$ & 19.563 & 13.683 & 15.845 & 22.013 & 21.589 & 11.938 & 9.456 & 17.114 & 16.153 \\
$70-74$ & 11.799 & 9.294 & 13.154 & 9.329 & 13.306 & 7.738 & 8.161 & 11.07 & 9.757 \\
$75+$ & 11.799 & 9.396 & 21.749 & 11.784 & 8.758 & 3.685 & 5.57 & 10 & 11.905 \\
Total & 100 & 100 & 100 & 100 & 100 & 100 & 100 & 100 & 100 \\
\hline
\end{tabular}


Other variables used in regression includes marriage, qualification for pensions, expenditure in one year, participation of health insurance, smoking and drinking habit, as well as BMI. Generally, a value of one means "yes" and zero means "no". Marriage is shown by variable "married" which is defined by question be 001 and measures the current marital status of respondents. Qualification for pensions are defined by questions fn069_w3 and fn002_w3 which measure respondents' qualification for resident's pension or other pension respectively. Expenditure in one year is defined by question ge 010 and is a sum of all expenditure a respondent has, and it can be used to infer the quality of life of the respondents. Participation of health insurance is defined by question ea001 and includes all kinds of insurances including urban employee medical insurance, urban resident medical insurance, new cooperative medial insurance, etc. Participation in any one of the insurances is counted. Smoking and drinking are defined by questions da059 and da067 respectively and they are a measurement of the respondents' lifestyle. BMI is calculated using the heights and weights of the respondents and it shows physical wellness. The summary statistics of the sample is shown in Table 3 below.

Table 3. Descriptive Statistics

\begin{tabular}{lllllll}
\hline Variable & Obs. & Mean & Std. Dev. & Min & Max & Missing \\
\hline Depression & 6162 & 1.894 & 1.0951 & 1 & 4 & 0 \\
total support & 6046 & 17113.65 & 900599 & 0 & $7.00 \mathrm{e}+07$ & 116 \\
visit & 5556 & 216.5495 & 315.297 & 0 & 2999 & 606 \\
male & 6162 & 0.5267 & 0.4993 & 0 & 1 & 0 \\
urban & 6162 & 0.3476 & 0.4762 & 0 & 1 & 0 \\
age & 6162 & 61.8698 & 9.3874 & 45 & 105 & 0 \\
education years & 6162 & 5.5945 & 4.1105 & 0 & 19 & 0 \\
married & 6162 & 0.8549 & 0.3522 & 0 & 1 & 0 \\
pension & 6162 & 0.7207 & 0.4487 & 0 & 1 & 0 \\
health insurance & 6162 & 0.968 & 0.1759 & 0 & 1 & 0 \\
total expenditure & 6162 & 13147.67 & 33069.4 & 0 & 806800 & 0 \\
smoking & 6162 & 0.3005 & 0.4583 & 0 & 1 & 0 \\
drinking & 6162 & 0.3693 & 0.4827 & 0 & 1 & 0 \\
BMI & 6162 & 23.9345 & 3.9269 & 13.7072 & 67.8895 & 0 \\
\hline
\end{tabular}

\section{RESULTS}

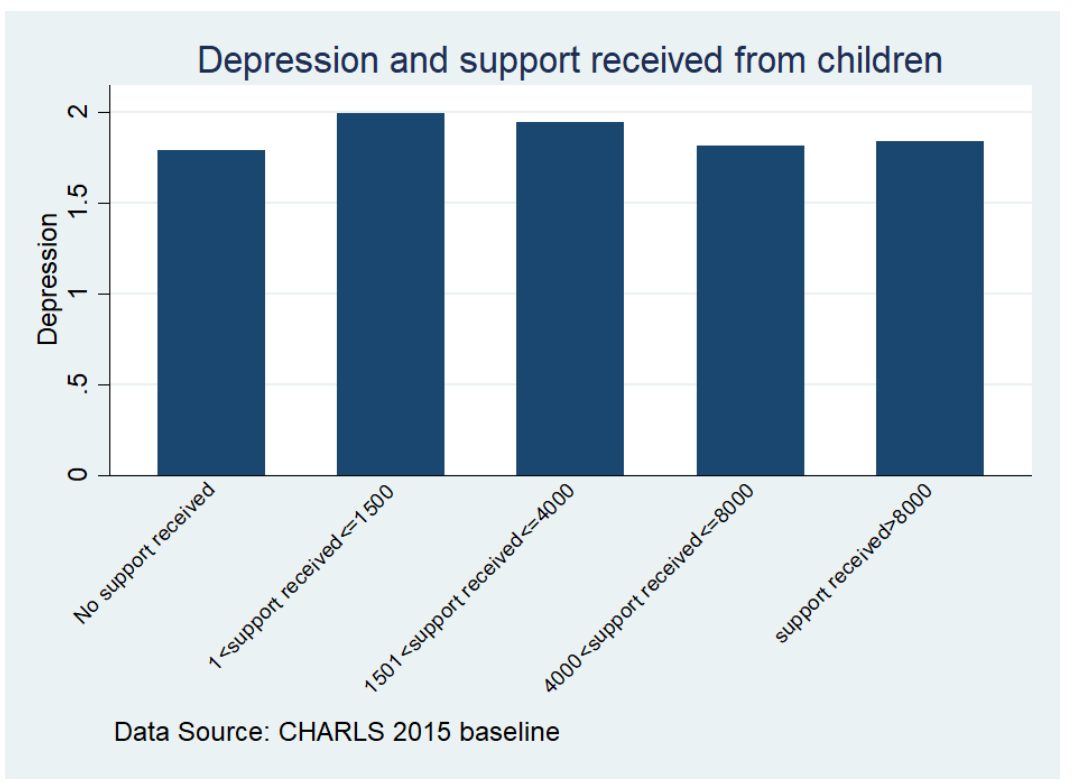

Fig. 4. Total support received from children and depression. In this histogram, $x$-axis represents the independent variable, total support from children, and y-axis represents level of depression of respondents. Depression is defined based on question DC011 
"I felt depressed". It is assessed by a four-point scale, scored as follows: $1=$ Rarely or none of the time ( $<1$ day), $2=$ Some or a little of the time (1-2 days), $3=$ Occasionally or a moderate amount of the time (3-4 days), and 4= Most or all of the time (5-7 days) out of a week. There are 5556 observations for this variable. The dependent variable, total support from children, comprises of money support and in-kind support and is defined by question CE009 "In the past one year, how much economic support did you and your spouse receive from your children?" It is measured in terms of monetary value but in this case we group them up to plot this graph. There are 6046 observations for this variable.

The relationship between depression and total support received from children is shown in Figure 4. The highest depression level of 2 are observed for respondents who received between 1 and 1500 yuan in the past year, and depression level decreases down the group to 1.75 for subsequent groups of respondents as money received increases from 1500 to maximum. However, the minimum level of depression (1.7) is reported by respondents who did not receive support at all. This suggests that not receiving monetary support from children do not necessarily lead to increase in depression level as other factors such as characteristic and personal experience may also play a part.

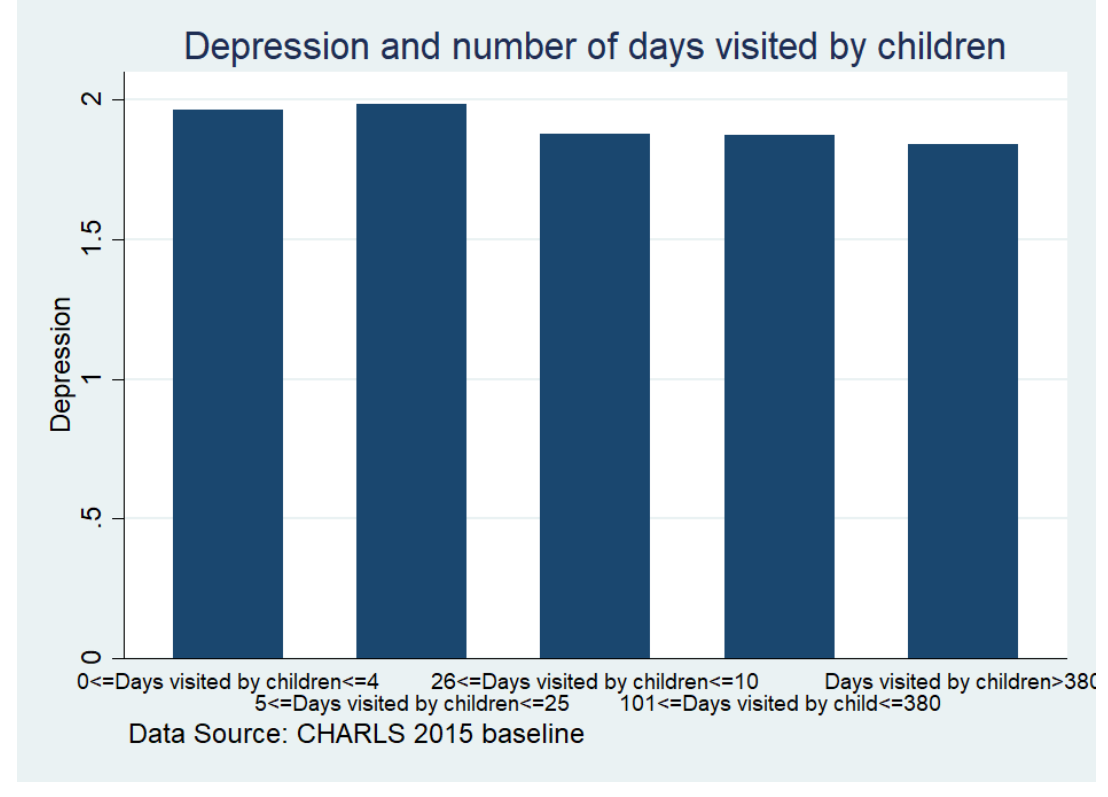

Fig. 5. Number of days visited by children and depression

The relationship between depression and frequency of visit is shown in Figure 5. An inverse relationship between frequency of visit of the child and depression of the respondents is observed. Respondents who were visited by their children for less than or equal to 25 days in last year have an average depression level of 2. Depression level then decreases from 2 to 1.8 as number of days visited by children increases from 26 to 380 and drops further to 1.7 for respondents who were visited for a total of more than 380 days by different children in a year. This shows that depression of elderly can be significantly reduced as number of days visited by children increases.

Table 4. Regression result for total support, frequency of visit and depression

\begin{tabular}{lll}
\hline & $(1)$ & $\begin{array}{l}(2) \\
\text { Depression \& } \\
\text { VARIABLES }\end{array}$ \\
\hline Total support received from children in a year & Depression \& Total Support & \\
Male & $-6.71 \mathrm{e}-09$ & \\
& $(1.52 \mathrm{e}-08)$ & $-0.346^{* * *}$ \\
Age & $-0.326^{* * *}$ & $(0.0379)$ \\
Years of Schooling & $(0.0361)$ & -0.00153 \\
& -0.00170 & $(0.00181)$ \\
Urban & $(0.00165)$ & $-0.0272^{* * *}$ \\
Married & $-0.0253^{* * *}$ & $(0.00399)$ \\
& $(0.00380)$ & $-0.190^{* * *}$ \\
& $-0.199 * * *$ & $(0.0319)$ \\
& $(0.0301)$ & 0.0118 \\
& 0.00916 & $(0.0448)$
\end{tabular}


Qualification for residents' and other pension

Total expenditure of last year

Current paricipation of health insurance

Smoking habit

Drinking habit

BMI

Total number of visits by adult children in past one year

Constant

Observations

R-squared

Standard errors in parentheses

$* * * \mathrm{p}<0.01, * * \mathrm{p}<0.05, * \mathrm{p}<0.1$

The regression outputs are shown in Table 4. According to the $\mathrm{F}$ test $(\mathrm{F}$-statistics $=28.90)$, the depression and total support model is significant, meaning that the model is correctly specified. The estimates show that the total support from children enters the depression regression equations in a negative manner and it is statistically insignificant ( $\mathrm{p}$-values $=0.659$ ). The following estimates are significant at a level of 0.01: male, years of schooling, and urban. Current participation of health insurance also has statistically significant coefficient ( $p$-values $<0.05$ ). Pension qualification have relatively less significant coefficient, ( $p$-values $<0.1$ ) but is nevertheless more significant than other variables, namely age, married, total expenditure, smoking habit, drinking habit and BMI. ( $\mathrm{p}$-value $>0.1$ )

According to the $\mathrm{F}$ test $(\mathrm{F}$-statistics $=28.25)$, the depression and times of visit model is also significant, meaning that the model is correctly specified. The estimates show that number of times of visit enters the depression regression equations in a negative manner and it is statistically significant ( $p$-values $=0.045<0.05$ ), suggesting that level of depression and frequency of visit by children are closely negatively related. The corresponding coefficients estimates are significant at a level of 0.01: male, years of schooling, urban, and current participation of health insurance. Qualification for residents' and other pension have statistically significant coefficient ( $p$-values $<0.05)$. Other variables, namely age, married, total expenditure, smoking habit, drinking habit and BMI, are statistically insignificant. (p-value $>0.1$ )

Intuitively, spending longer time with children lowers the depression level of elderly and this is especially true for female elderly living in rural areas, who do not have healthcare or pension and have low level of education background. Total monetary support, on the other hand, plays a less pivotal role in reducing depression level of the elderly.

\begin{tabular}{ll}
$-0.0598^{*}$ & $-0.0812 * *$ \\
$(0.0306)$ & $(0.0320)$ \\
$-6.32 \mathrm{e}-07$ & $-5.45 \mathrm{e}-07$ \\
$(4.14 \mathrm{e}-07)$ & $(4.77 \mathrm{e}-07)$ \\
$-0.193 * *$ & $-0.234 * *$ \\
$(0.0802)$ & $(0.0859)$ \\
0.0270 & 0.0284 \\
$(0.0352)$ & $(0.0367)$ \\
-0.0426 & -0.0282 \\
$(0.0314)$ & $(0.0329)$ \\
-0.00558 & -0.00617 \\
$(0.00362)$ & $(0.00378)$ \\
& $-9.68 \mathrm{e}-05^{* *}$ \\
$2.749 * * *$ & $(4.82 \mathrm{e}-05)$ \\
$(0.173)$ & $2.850^{* * *}$ \\
& $(0.186)$ \\
6,046 & 5,556 \\
0.054 & 0.058 \\
\hline
\end{tabular}

\section{DISCUSSION}

Our regression results show a statistically significant negative association between number of days the respondents see their children in a year and their depression level. However, the association between depression level and total support (sum of monetary and in-kind support) is not statistically significant. This indicates that whether or not the elderly is together physically with children frequently is a much stronger factor that lowers elderly's depression level. Monetary support, on the other hand, does not achieve this goal as elderly (especially those who live alone) usually desire accompany instead of money, so getting more wealthy does not necessarily make them much happier. This is especially true given the hectic work schedule and busy lifestyle that working adults have in China and as a result, these adults are deprived of time which should have been spent with their parents. Visiting their parents usually means travelling long distance from city to their hometown and many are not dedicated to do so due to financial reasons. As some sorts of compensation, many think of sending their parents more money to allow them live a more luxurious life style in their old ages but sometimes this may not work well as shown by this study, this might because the elderly would rather want a family dinner rather than a ten-thousand-dollar transaction.

Other research has highlighted similar results. According to research conducted among elderly in Spain, low emotional support and reception of instrumental aid were significantly associated with poor self-reported health of elderly. Moreover, depressive symptoms were associated with low emotional support, reception of instrumental help and being a widower who did not share living arrangements with children. (MV Zunzunegui, 2001) Similarly, a study in India shows that Both late-life mental disorders (depression and dementia) were attributed to abuse, neglect, or lack of love 
on the part of children towards a parent. (Vikram Patel, 2001) On the other hand, other research highlighted that lower living standards are both independently related to mental health and also contribute to diminished opportunities for social support. Social support and loneliness in turn, are related to mental health, proving that although extra wealth does not significantly lower depression rate of the elderly, basic standard of living should not be too low for good mental health of the old. Hence, elderly should be supported both socially and economically for healthy mindsets.

Given the high risk of physical problems arising from mental problems, cognitive issues of elderly should not be neglected so as to give the elderly happiness and fulfilling old age they deserve. Simple things like a regular visit can make a huge different in an elderly's life, and they are preferred over large sum of money transaction by the elderly. The general trend towards people living longer and being healthier for longer means that the main emphasize in terms of assistance and support should be put towards the 'older' aged that is those over 75 or 80 years of age. Given the need to offer more care to a person for the last few years of his or her life, government should implement policies that enable workers with parents aged 75 and above to devote more time to accompanying their parents. This include longer weekends and holidays as well as shorter working hours per day. This is already implemented in cities including Fujian, Guangxi, Hubei, Hainan, Heilongjiang and Guangzhou. Other cities should follow suit and take action towards allowing people to have more spare time to be spent with their parents. Direct subsidies should also be given to people with parents older than 75 to ensure that these people can afford to work shorter hours and pay their parents more visit but still be able to have sufficient disposable income. With more time and monetary means, hopefully spending time with the elderly will be a commitment that all adults are give and caring for the elderly will no longer be merely a token symbol.

\section{CONCLUSION}

In this paper, we studied the relationship between physical and monetary support from children and mental wellbeing of the elderly in China using a new sample of middle-aged and older Chinese respondents. Modeled after the American HRS, the CHARLS survey respondents are 45 years and older in 28 provinces in China. Our work on childhood health in CHARLS relied on two complementary measures that proxy for support that elderly received from their adult children. The first is a statement of monetary value received from children in the past one year. The second is frequency of visit by children for the past one year in terms of number of days. We related both variables to level of depression of respondents, which reflects their mental health. We find a statistically significant negative association between frequency of visit and level of depression particularly among women who live in rural areas, who do not have high level of education, and not participants of pension or health insurance. Monetary support, however, does not have a significant relation with depression level.

\section{REFERENCES}

[1] Charls, 2016. "About CHARLS". Available at:

[2] http://charls.pku.edu.cn/en/page/about/charls.

[3] Fan and Fang, 2018. "Living arrangements of the elderly: A new perspective from choice constraints in China." China Economic Review 50 (2018) 101-116.

[4] Geldsetzer and Haakenstad, 2018. "Non-technical health care quality and health system responsiveness in middle-income countries: a corss-sectional study in China, Ghana, India, Mexico, Russia, and South Africa." Journal of global health. Vol.8 No.2. 020417.

[5] Guangming $\mathrm{Xu}$, Gong Chen, Qin Zhou, et al. "Prevalence of Mental Disorders among Older Chinese People in Tianjin City." Can J Psychiatry. 2017 Nov; 62(11): 778-786.

[6] Kate B et al,2010. "Anxiety disorder in older adults: a comprehensive review." Wiley Online Library. Published online.

[7] Kesavayuth and Liang, 2018. "An Active Lifestyle and Cognitive Function: Evidence from China." Journal of the Economics of Ageing. May 2018.

[8] Larry C.Mullins \& Elizabeth Dugan, 1991. "Elderly Social Relationships with Adult children and Close Friends and Depression.” Department of Gerontology, College of Arts and Science. In Journal of Social Behaviour and Personality, 1991, Vol.6, No.2, 315-328

[9] Lin and Li, 2016. "The impact of ambient air pollution on suicide mortality: a case-crossover study in Guangzhou, China" In Environmental Health. 201615:90

[10] Patel and Prince, 2001. "Aging and mental health in a developing country: Who cares? Qualitative studies from Goa, India." Cambridge university press. Published online.

[11] Rapoza K, 2017. "China's aging population becoming more of a problem." Avail-able at:

[12] https://www. forbes. com/sites/kenrapoza/2017/02/21/chinas-aging-popula tion-becoming-more-of-a-problem.

[13] Wu and Dong, 2018. "Financial transfers from adult children and depressive symptoms among mid-aged and elderly residents in China - evidence from the China health and retirement longitudinal study" BMC Public Health 201818:882 
[14] Zhang and Liu, 2016. "Relationship between body mass index and depressive symptoms: the 'fat and jolly' hypothesis for the middle-aged and elderly in China" BMC Public Health BMC series - open, inclusive and trusted 201616:1201

[15] Zunzunegui and Béland, 2001. "Support from children, living arrangements, self-rated health and depressive symptoms of older people in Spain" International Journal of Epidemiology, Volume 30, Issue 5, Pages 1090-1099 